CRYSTALLOGRAPHIC COMMUNICATIONS

ISSN 2056-9890

Received 16 March 2015

Accepted 22 March 2015

Edited by W. T. A. Harrison, University of Aberdeen, Scotland

Keywords: crystal structure; crystal packing; quinoline; carbazole; 9-ethyl-9H-carbazol-3amine; $\mathrm{C}-\mathrm{H} \cdots \pi$ interactions; $\pi-\pi$ interactions

CCDC reference: 1027676

Supporting information: this article has supporting information at journals.iucr.org/e

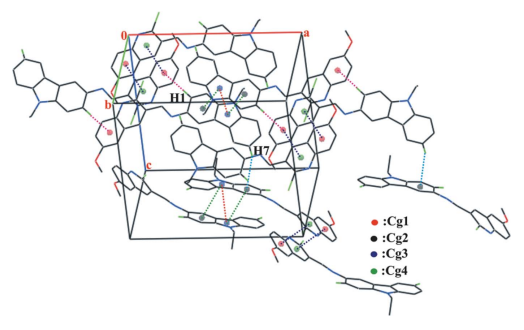

OPEN $\odot$ ACCESS

\section{Crystal structure of $(E)-\mathrm{N}$-[(2-chloro-6-methoxy- quinolin-3-yl)methylidene]-9-ethyl-9H-carbazol-3- amine}

Kannan Thirumurthy, ${ }^{a}$ Ganesamoorthy Thirunarayanan ${ }^{\mathrm{a} *}$ and S. Murugavel ${ }^{\mathrm{b} *}$

a Department of Chemistry, Annamalai University, Annamalainagar 608 002, Chidambaram, Tamilnadu, India, and

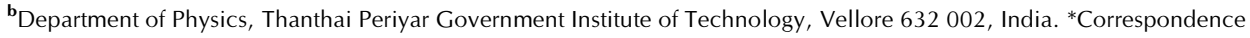
e-mail: drgtnarayanan@rediffmail.com,smurugavel27@gmail.com

In the title compound, $\mathrm{C}_{25} \mathrm{H}_{20} \mathrm{ClN}_{3} \mathrm{O}$, the $\mathrm{C}=\mathrm{N}$ bond of the central imine group adopts an $E$ conformation. The mean planes through the essentially planar carbazole [maximum deviation $=0.052(2) \AA$ ] and quinoline [maximum deviation $=0.050$ (2) $\AA$ ] ring systems form a dihedral angle of $50.2(1)^{\circ}$. In the crystal, molecules are linked by $\mathrm{C}-\mathrm{H} \cdots \pi$ and $\pi-\pi$ interactions [centroidcentroid distances ranging from 3.635 (2) to 3.739 (2) $\AA$ ], forming a threedimensional supramolecular network.

\section{Chemical context}

It has been reported that carbazole derivatives possess various biological activities, such as antitumor (Itoigawa et al., 2000), anti-oxidative (Tachibana et al., 2001), anti-inflammatory and antimutagenic (Ramsewak et al., 1999). Carbazole derivatives also exhibit electroactivity and luminescence properties and are considered to be potential candidates for electronic devices such as colour displays, organic semiconductor lasers and solar cells (Friend et al., 1999). These compounds are thermally and photochemically stable, which makes them useful materials for technological applications: for instance, the carbazole ring is easily funtionalized and covalently linked to other molecules (Díaz et al., 2002). This enables its use as a convenient building block for the design and synthesis of molecular glasses, which are widely studied as components of electroactive and photoactive materials (Zhang et al., 2004). Quinoline derivatives are known to possess a variety of biological properties such as antimalarial and antiviral activity (Cunico et al., 2006; Hartline et al., 2005). Against this background, and in order to obtain detailed information on its molecular conformation in the solid state, the crystal structure of the title compound has been determined.

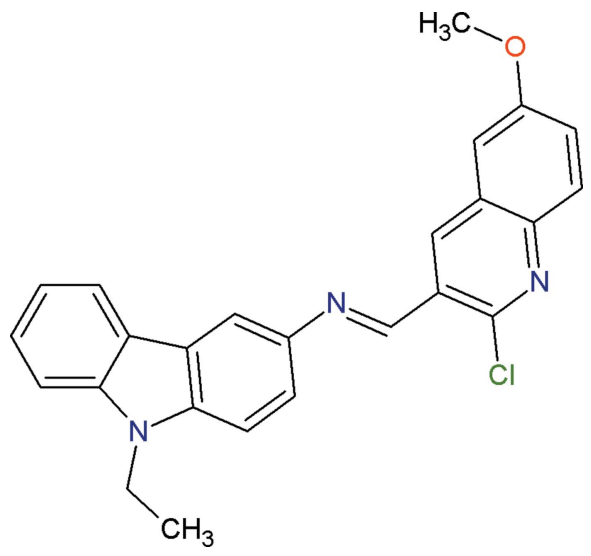




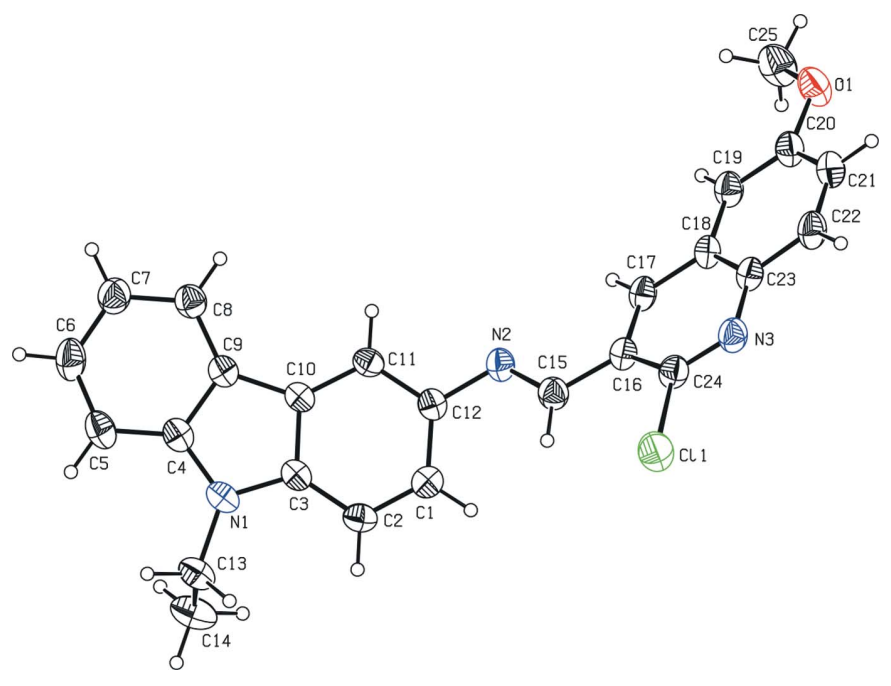

Figure 1

Molecular structure of the title compound showing displacement ellipsoids at the $30 \%$ probability level. $\mathrm{H}$ atoms are drawn as a small spheres of arbitrary radii.

\section{Structural commentary}

Fig. 1. shows a displacement ellipsoid plot of (I), with the atom-numbering scheme. The $\mathrm{C}=\mathrm{N}$ bond of the central imine group adopts an $E$ conformation. The mean planes through the essentially planar carbazole [N1/C1-C12; maximum deviation $=0.052(2) \AA$ for atom $\mathrm{C} 12]$ and quinoline $[\mathrm{N} 3 / \mathrm{C} 16-$ C24; maximum deviation $=0.050$ (2) $\AA$ for atom C16] ring systems form a dihedral angle of $50.2(1)^{\circ}$. The sum of the bond angles around $\mathrm{N} 1\left(360.05^{\circ}\right)$ of the pyrrole ring is in accordance with $s p^{2}$ hybridization. Atom Cl1 deviates from the plane of the attached quinoline ring system by 0.100 (1) $\AA$. The geometric parameters of the title molecule agree well with those reported for similar structures (Murugavel et al., 2009; Archana et al., 2011).

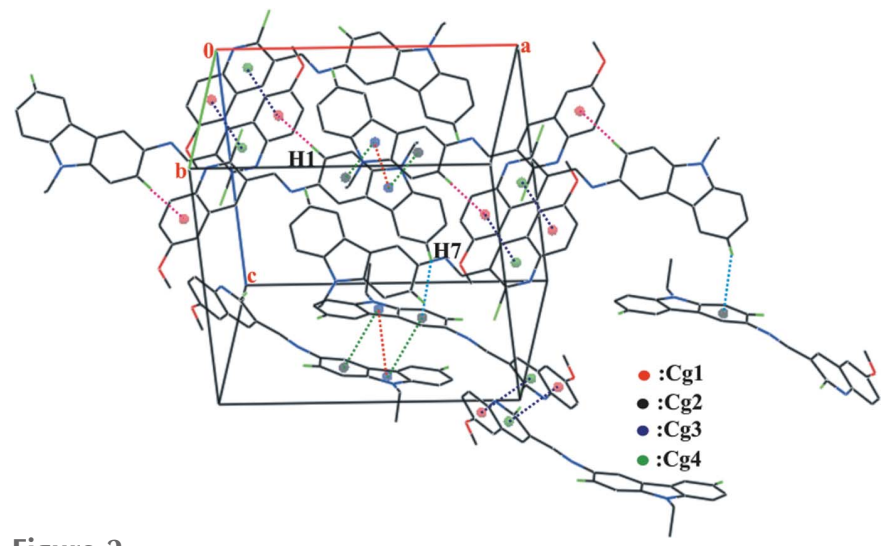

Figure 2

Part of the crystal structure of the title compound showing the $\mathrm{C}-\mathrm{H} \cdots \pi$ and $\pi-\pi$ interactions, which lead to the formation of a three-dimensional supramolecular network. Hydrogen atoms not involved in hydrogen bonding are omitted for clarity. $C g 1, C g 2, C g 3$ and $C g 4$ are the centroids of the $\mathrm{C} 18-\mathrm{C} 23$ benzene ring, the $\mathrm{C} 1-\mathrm{C} 3 / \mathrm{C} 10-\mathrm{C} 12$ benzene ring, the $\mathrm{N} 1 /$ $\mathrm{C} 3 / \mathrm{C} 4 / \mathrm{C} 9 / \mathrm{C} 10$ pyrrole ring and the $\mathrm{N} 3 / \mathrm{C} 16-\mathrm{C} 18 / \mathrm{C} 23 / \mathrm{C} 24$ pyridine ring, respectively.
Table 1

Hydrogen-bond geometry $\left(\AA,^{\circ}\right)$.

$C g 1$ and $C g 2$ are the centroids of the $\mathrm{C} 18-\mathrm{C} 23$ and $\mathrm{C} 1-\mathrm{C} 3 / \mathrm{C} 10-\mathrm{C} 12$ benzene rings, respectively.

\begin{tabular}{lllll}
\hline$D-\mathrm{H} \cdots A$ & $D-\mathrm{H}$ & $\mathrm{H} \cdots A$ & $D \cdots A$ & $D-\mathrm{H} \cdots A$ \\
\hline $\mathrm{C} 1-\mathrm{H} 1 \cdots C g 1^{\mathrm{i}}$ & 0.93 & 2.87 & $3.718(3)$ & 152 \\
$\mathrm{C} 7-\mathrm{H} 7 \cdots C g 2^{\mathrm{ii}}$ & 0.93 & 2.97 & $3.688(2)$ & 145
\end{tabular}

Symmetry codes: (i) $-x,-y,-z+1$; (ii) $-x+1, y+\frac{1}{2},-z+\frac{3}{2}$.

\section{Supramolecular features}

In the crystal, molecules are linked by two $\mathrm{C}-\mathrm{H} \cdots \pi$ interactions; the first one between the benzene $\mathrm{H}$ atom of the carbazole ring system and the benzene ring of an adjacent molecule, with a $\mathrm{C} 1-\mathrm{H} 1 \cdots C g 1^{\mathrm{i}}$ and the second one between the benzene $\mathrm{H}$ atom of the carbazole ring system and the benzene ring of an adjacent molecule, with a $\mathrm{C} 7-\mathrm{H} 7 \cdots \mathrm{Cg}^{2}$. The molecules are further linked by $\pi-\pi$ interactions with $C g 3-C g 3^{\mathrm{iii}}, C g 3-C g 2^{\mathrm{iii}}, C g 2-C g 3^{\mathrm{iii}}, C g 4-C g 1^{\mathrm{iv}}$ and $C g 1-C g 4^{\mathrm{iv}}$ separations of $3.735(2), 3.739$ (2), $3.739(2), 3.635$ (2) and 3.635 (2) $\AA$, respectively, forming a three-dimensional supramolecular network (Table 1 and Fig. 2; $C g 1, C g 2, C g 3$ and $C g 4$ are the centroids of $\mathrm{C} 18-\mathrm{C} 23$ benzene ring, the $\mathrm{C} 1-\mathrm{C} 3 / \mathrm{C} 10$ $\mathrm{C} 12$ benzene ring, the $\mathrm{N} 1 / \mathrm{C} 3 / \mathrm{C} 4 / \mathrm{C} 9 / \mathrm{C} 10$ pyrrole ring and the $\mathrm{N} 3 / \mathrm{C} 16-\mathrm{C} 18 / \mathrm{C} 23 / \mathrm{C} 24$ pyridine ring, respectively; symmetry codes: (i) $-x,-y, 1-z$; (ii) $1-x, \frac{1}{2}+y, \frac{3}{2}-z$; (iii) $1-x,-y$, $1-z$ and (iv) $-x, 1-y, 1-z$ ).

Table 2

Experimental details.

\begin{tabular}{|c|c|}
\hline \multicolumn{2}{|l|}{ Crystal data } \\
\hline Chemical formula & $\mathrm{C}_{25} \mathrm{H}_{20} \mathrm{ClN}_{3} \mathrm{O}$ \\
\hline$M_{\mathrm{r}}$ & 413.89 \\
\hline Crystal system, space group & Monoclinic, $P 2_{1} / c$ \\
\hline Temperature $(\mathrm{K})$ & 293 \\
\hline$a, b, c(\AA)$ & $15.060(3), 8.8231(15), 15.332(3)$ \\
\hline$\beta\left(^{\circ}\right)$ & $93.344(3)$ \\
\hline$V\left(\mathrm{~A}^{3}\right)$ & $2033.9(6)$ \\
\hline$Z$ & 4 \\
\hline Radiation type & Mo $K \alpha$ \\
\hline$\mu\left(\mathrm{mm}^{-1}\right)$ & 0.21 \\
\hline Crystal size $(\mathrm{mm})$ & $0.24 \times 0.21 \times 0.16$ \\
\hline \multicolumn{2}{|l|}{ Data collection } \\
\hline Diffractometer & Bruker SMART CCD \\
\hline Absorption correction & $\begin{array}{l}\text { Multi-scan (SADABS; Sheldrick, } \\
\text { 1996) }\end{array}$ \\
\hline$T_{\min }, T_{\max }$ & $0.951,0.967$ \\
\hline $\begin{array}{l}\text { No. of measured, independent and } \\
\text { observed }[I>2 \sigma(I)] \text { reflections }\end{array}$ & $20538,4023,2340$ \\
\hline$R_{\text {int }}$ & 0.056 \\
\hline$(\sin \theta / \lambda)_{\max }\left(\AA^{-1}\right)$ & 0.620 \\
\hline \multicolumn{2}{|l|}{ Refinement } \\
\hline$R\left[F^{2}>2 \sigma\left(F^{2}\right)\right], w R\left(F^{2}\right), S$ & $0.051,0.133,1.01$ \\
\hline No. of reflections & 4023 \\
\hline No. of parameters & 273 \\
\hline $\mathrm{H}$-atom treatment & H-atom parameters constrained \\
\hline$\Delta \rho_{\max }, \Delta \rho_{\min }\left(\mathrm{e} \AA^{-3}\right)$ & $0.24,-0.14$ \\
\hline
\end{tabular}

Computer programs: SMART and SAINT (Bruker, 2002), SHELXS97 and SHELXL97 (Sheldrick, 2008), ORTEP-3 for Windows (Farrugia (1997) and PLATON (Spek, 2009) 


\section{Synthesis and crystallization}

A $25 \mathrm{ml}$ round-bottom flask was charged with dimedone (1 mmol), 2-chloro-6-methoxyquinoline-3-carbaldehyde (1 mmol) 9-ethyl-9H-carbazol-3-amine $(1 \mathrm{mmol})$ and sulfated $\mathrm{SnO}_{2}$-fly ash catalyst $(50 \mathrm{mg})$ in water $(15 \mathrm{ml})$ and was refluxed at $353 \mathrm{~K}$ for $5-10$ minutes. The completion of the reaction was monitored by TLC (ethyl acetate and hexane as an eluent $20 \%$ ). After completion, the reaction mixture was cooled to ambient temperature. Then dichloromethane $(20 \mathrm{ml})$ was added and the organic layer filtered, dried on anhydrous $\mathrm{Na}_{2} \mathrm{SO}_{4}$ and the solvent removed using a rotary evaporator. The crude product was purified by column chromatography on silica gel (200 mesh) with hexane and ethyl acetate (4:1) as eluent to afford the title compound in good yield $(10 \%)$. Red blocks suitable for X-ray diffraction analysis were obtained by recrystallization from dichloromethane solution at room temperature.

\section{Refinement}

Crystal data, data collection and structure refinement details are summarized in Table 2. $\mathrm{H}$ atoms were positioned geometrically and constrained to ride on their parent atom with $\mathrm{C}-$ $\mathrm{H}=0.93-0.97 \AA$ and with $U_{\text {iso }}(\mathrm{H})=1.5 U_{\text {eq }}$ for methyl $\mathrm{H}$ atoms and $1.2 U_{\text {eq }}(\mathrm{C})$ for other $\mathrm{H}$ atoms.

\section{Acknowledgements}

The authors thank Professor M. Periasamy, School of Chemistry, University of Hyderabad, for providing laboratory facilities and are also grateful to the UGC Networking Resource Centre, School of Chemistry, University of Hyderabad, for providing characterization facilities.

\section{References}

Archana, R., Yamuna, E., Rajendra Prasad, K. J., Thiruvalluvar, A. \& Butcher, R. J. (2011). Acta Cryst. E67, o1799.

Bruker (2002). SMART and SAINT. Bruker AXS Inc., Madison, Wisconsin, U. S. A.

Cunico, W., Cechinel, C. A., Bonacorso, H. G., Martins, M. A. P., Zanatta, N., de Souza, M. V. N., Freitas, I. O., Soares, R. P. P. \& Krettli, A. U. (2006). Bioorg. Med. Chem. Lett. 16, 649-653.

Díaz, J. L., Villacampa, B., López-Calahorra, F. \& Velasco, D. (2002). Chem. Mater. 14, 2240-2251.

Farrugia, L. J. (1997). J. Appl. Cryst. 30, 565.

Friend, R. H., Gymer, R. W., Holmes, A. B., Burroughes, J. H., Marks, R. N., Taliani, C., Bradley, D. D. C., Dos Santos, D. A., Brédas, J. L., Lögdlund, M. \& Salaneck, W. R. (1999). Nature, 397, 121-128.

Hartline, C. B., Harden, E. A., Williams-Aziz, S. L., Kushner, N. L., Brideau, R. J. \& Kern, E. R. (2005). Antiviral Res. 65, 97-105.

Itoigawa, M., Kashiwada, Y., Ito, C., Furukawa, H., Tachibana, Y., Bastow, K. F. \& Lee, K. H. (2000). J. Nat. Prod. 63, 893-897.

Murugavel, S., Ranjith, S., SubbiahPandi, A., Periyasami, G. \& Raghunathan, R. (2009). Acta Cryst. E65, o139-o140.

Ramsewak, R. S., Nair, M. G., Strasburg, G. M., DeWitt, D. L. \& Nitiss, J. L. (1999). J. Agric. Food Chem. 47, 444-447.

Sheldrick, G. M. (1996). SADABS. University of Göttingen, Germany.

Sheldrick, G. M. (2008). Acta Cryst. A64, 112-122.

Spek, A. L. (2009). Acta Cryst. D65, 148-155.

Tachibana, Y., Kikuzaki, H., Lajis, N. H. \& Nakatani, N. (2001). J. Agric. Food Chem. 49, 5589-5594.

Zhang, Q., Chen, J., Cheng, Y., Wang, L., Ma, D., Jing, X. \& Wang, F. (2004). J. Mater. Chem. 14, 895-900. 


\section{supporting information}

Acta Cryst. (2015). E71, 421-423 [doi:10.1107/S2056989015005794]

\section{Crystal structure of (E)-N-[(2-chloro-6-methoxyquinolin-3-yl)methylidene]-9- ethyl-9H-carbazol-3-amine}

\section{Kannan Thirumurthy, Ganesamoorthy Thirunarayanan and S. Murugavel}

Computing details

Data collection: SMART (Bruker, 2002); cell refinement: SAINT (Bruker, 2002); data reduction: SAINT (Bruker, 2002); program(s) used to solve structure: SHELXS97 (Sheldrick, 2008); program(s) used to refine structure: SHELXL97 (Sheldrick, 2008); molecular graphics: ORTEP-3 for Windows (Farrugia (1997); software used to prepare material for publication: SHELXL97 (Sheldrick, 2008) and PLATON (Spek, 2009).

(E)-N-[(2-chloro-6-methoxyquinolin-3-yl)methylidene]-9-ethyl-9H-carbazol-3-amine

\section{Crystal data}

$\mathrm{C}_{25} \mathrm{H}_{20} \mathrm{ClN}_{3} \mathrm{O}$

$M_{r}=413.89$

Monoclinic, $P 2{ }_{1} / c$

Hall symbol: -P $2 \mathrm{ybc}$

$a=15.060(3) \AA$

$b=8.8231(15) \AA$

$c=15.332(3) \AA$

$\beta=93.344(3)^{\circ}$

$V=2033.9(6) \AA^{3}$

$Z=4$

\section{Data collection}

\section{Bruker SMART CCD}

diffractometer

Radiation source: fine-focus sealed tube

Graphite monochromator

$\omega$ scans

Absorption correction: multi-scan

(SADABS; Sheldrick, 1996)

$T_{\min }=0.951, T_{\max }=0.967$

\section{Refinement}

Refinement on $F^{2}$

Least-squares matrix: full

$R\left[F^{2}>2 \sigma\left(F^{2}\right)\right]=0.051$

$w R\left(F^{2}\right)=0.133$

$S=1.01$

4023 reflections

273 parameters

0 restraints
$F(000)=864$

$D_{\mathrm{x}}=1.352 \mathrm{Mg} \mathrm{m}^{-3}$

Mo $K \alpha$ radiation, $\lambda=0.71073 \AA$

Cell parameters from 4051 reflections

$\theta=1.4-26.1^{\circ}$

$\mu=0.21 \mathrm{~mm}^{-1}$

$T=293 \mathrm{~K}$

Block, red

$0.24 \times 0.21 \times 0.16 \mathrm{~mm}$

20538 measured reflections

4023 independent reflections

2340 reflections with $I>2 \sigma(I)$

$R_{\text {int }}=0.056$

$\theta_{\max }=26.1^{\circ}, \theta_{\min }=1.4^{\circ}$

$h=-18 \rightarrow 18$

$k=-10 \rightarrow 10$

$l=-18 \rightarrow 18$

Primary atom site location: structure-invariant direct methods

Secondary atom site location: difference Fourier map

Hydrogen site location: inferred from neighbouring sites

$\mathrm{H}$-atom parameters constrained 
$w=1 /\left[\sigma^{2}\left(F_{\mathrm{o}}^{2}\right)+(0.0565 P)^{2}+0.145 P\right]$

where $P=\left(F_{\mathrm{o}}^{2}+2 F_{\mathrm{c}}{ }^{2}\right) / 3$

$(\Delta / \sigma)_{\max }<0.001$

$$
\begin{aligned}
& \Delta \rho_{\max }=0.24 \mathrm{e} \AA^{-3} \\
& \Delta \rho=-0.14 \mathrm{e} \AA^{-3}
\end{aligned}
$$

$\Delta \rho_{\min }=-0.14 \mathrm{e} \AA^{-3}$

\section{Special details}

Geometry. All esds (except the esd in the dihedral angle between two 1.s. planes) are estimated using the full covariance matrix. The cell esds are taken into account individually in the estimation of esds in distances, angles and torsion angles; correlations between esds in cell parameters are only used when they are defined by crystal symmetry. An approximate (isotropic) treatment of cell esds is used for estimating esds involving 1.s. planes.

Refinement. Refinement of $\mathrm{F}^{2}$ against ALL reflections. The weighted $\mathrm{R}$-factor $\mathrm{wR}$ and goodness of fit $\mathrm{S}$ are based on $\mathrm{F}^{2}$, conventional R-factors $R$ are based on $F$, with $F$ set to zero for negative $F^{2}$. The threshold expression of $F^{2}>2$ sigma $\left(F^{2}\right)$ is used only for calculating R-factors(gt) etc. and is not relevant to the choice of reflections for refinement. R-factors based on $\mathrm{F}^{2}$ are statistically about twice as large as those based on F, and R-factors based on ALL data will be even larger.

Fractional atomic coordinates and isotropic or equivalent isotropic displacement parameters $\left(\AA^{2}\right)$

\begin{tabular}{|c|c|c|c|c|}
\hline & $x$ & $y$ & $z$ & $U_{\text {iso }} * / U_{\text {eq }}$ \\
\hline $\mathrm{C} 11$ & $0.06066(5)$ & $0.06058(9)$ & $0.30880(4)$ & $0.0824(3)$ \\
\hline $\mathrm{C} 10$ & $0.43767(14)$ & $0.0240(2)$ & $0.62074(13)$ & $0.0455(6)$ \\
\hline $\mathrm{C} 11$ & $0.35381(15)$ & $0.0892(3)$ & $0.61516(14)$ & $0.0481(6)$ \\
\hline H11 & 0.3441 & 0.1814 & 0.6424 & $0.058^{*}$ \\
\hline $\mathrm{N} 2$ & $0.20216(12)$ & $0.0956(2)$ & $0.55982(12)$ & $0.0541(5)$ \\
\hline $\mathrm{O} 1$ & $-0.20036(11)$ & $0.5826(2)$ & $0.63150(12)$ & $0.0760(6)$ \\
\hline $\mathrm{C} 23$ & $-0.08229(15)$ & $0.3293(3)$ & $0.44161(15)$ & $0.0531(6)$ \\
\hline N3 & $-0.04814(13)$ & $0.2485(2)$ & $0.37581(12)$ & $0.0585(5)$ \\
\hline N1 & $0.53829(13)$ & $-0.1591(2)$ & $0.59420(13)$ & $0.0562(5)$ \\
\hline $\mathrm{C} 9$ & $0.52392(15)$ & 0.0703 & $0.65834(14)$ & $0.0484(6)$ \\
\hline $\mathrm{C} 3$ & $0.45006(15)$ & $-0.1178(3)$ & $0.58178(15)$ & $0.0491(6)$ \\
\hline $\mathrm{C} 12$ & $0.28459(15)$ & $0.0167(3)$ & $0.56899(14)$ & $0.0497(6)$ \\
\hline $\mathrm{C} 4$ & $0.58357(16)$ & $-0.0465(3)$ & $0.64053(15)$ & $0.0524(6)$ \\
\hline $\mathrm{C} 2$ & $0.38021(16)$ & $-0.1946(3)$ & $0.53925(15)$ & $0.0566(6)$ \\
\hline $\mathrm{H} 2$ & 0.3885 & -0.2902 & 0.5155 & $0.068 *$ \\
\hline $\mathrm{C} 24$ & $0.02130(16)$ & $0.1662(3)$ & $0.39528(15)$ & $0.0555(6)$ \\
\hline C18 & $-0.04726(14)$ & $0.3209(3)$ & $0.52873(15)$ & $0.0494(6)$ \\
\hline C19 & $-0.08694(15)$ & $0.4038(3)$ & $0.59474(15)$ & $0.0576(6)$ \\
\hline H19 & -0.0646 & 0.3964 & 0.6524 & $0.069 *$ \\
\hline $\mathrm{C} 16$ & $0.06697(14)$ & $0.1538(3)$ & $0.47843(15)$ & $0.0509(6)$ \\
\hline $\mathrm{C} 20$ & $-0.15802(16)$ & $0.4946(3)$ & $0.57397(16)$ & $0.0583(7)$ \\
\hline $\mathrm{C} 1$ & $0.29845(16)$ & $-0.1263(3)$ & $0.53286(15)$ & $0.0569(6)$ \\
\hline $\mathrm{H} 1$ & 0.2510 & -0.1762 & 0.5038 & $0.068 *$ \\
\hline $\mathrm{C} 22$ & $-0.15569(16)$ & $0.4262(3)$ & $0.42313(17)$ & $0.0634(7)$ \\
\hline $\mathrm{H} 22$ & -0.1796 & 0.4345 & 0.3660 & $0.076^{*}$ \\
\hline $\mathrm{C} 8$ & $0.55487(16)$ & $0.1987(3)$ & $0.70283(15)$ & $0.0590(7)$ \\
\hline H8 & 0.5159 & 0.2760 & 0.7161 & $0.071^{*}$ \\
\hline $\mathrm{C} 17$ & $0.02964(14)$ & 0.2319 & $0.54418(15)$ & $0.0534(6)$ \\
\hline $\mathrm{H} 17$ & 0.0558 & 0.2260 & 0.6006 & $0.064 *$ \\
\hline $\mathrm{C} 15$ & $0.15054(15)$ & 0.0715 & $0.49281(16)$ & $0.0566(6)$ \\
\hline H15 & 0.1664 & -0.0001 & 0.4520 & $0.068 *$ \\
\hline C6 & $0.70181(18)$ & $0.0939(4)$ & $0.70840(17)$ & $0.0737(8)$ \\
\hline
\end{tabular}




$\begin{array}{lllll}\text { H6 } & 0.7618 & 0.1043 & 0.7252 & 0.088^{*} \\ \text { C13 } & 0.57753(17) & -0.2968(3) & 0.56101(16) & 0.0649(7) \\ \text { H13A } & 0.6399 & -0.2785 & 0.5522 & 0.078^{*} \\ \text { H13B } & 0.5483 & -0.3215 & 0.5048 & 0.078^{*} \\ \text { C21 } & -0.19161(16) & 0.5068(3) & 0.48695(17) & 0.0635(7) \\ \text { H21 } & -0.2392 & 0.5712 & 0.4731 & 0.076^{*} \\ \text { C5 } & 0.67368(17) & -0.0363(3) & 0.66605(16) & 0.0645(7) \\ \text { H5 } & 0.7131 & -0.1142 & 0.6549 & 0.077^{*} \\ \text { C7 } & 0.64419(17) & 0.2099(3) & 0.72704(17) & 0.0691(7) \\ \text { H7 } & 0.6658 & 0.2960 & 0.7561 & 0.083^{*} \\ \text { C25 } & -0.17246(19) & 0.5688(4) & 0.72159(18) & 0.0863(9) \\ \text { H25A } & -0.1805 & 0.4660 & 0.7402 & 0.129^{*} \\ \text { H25B } & -0.2073 & 0.6352 & 0.7555 & 0.129^{*} \\ \text { H25C } & -0.1108 & 0.5957 & 0.7297 & 0.129^{*} \\ \text { C14 } & 0.5698(2) & -0.4287(3) & 0.62109(19) & 0.0851(9) \\ \text { H14A } & 0.6001 & -0.4060 & 0.6764 & 0.128^{*} \\ \text { H14B } & 0.5963 & -0.5164 & 0.5961 & 0.128^{*} \\ \text { H14C } & 0.5082 & -0.4485 & 0.6293 & 0.128^{*}\end{array}$

Atomic displacement parameters $\left(\AA^{2}\right)$

\begin{tabular}{lllllll}
\hline & $U^{11}$ & $U^{22}$ & $U^{33}$ & $U^{12}$ & $U^{13}$ & $U^{23}$ \\
\hline C11 & $0.0740(5)$ & $0.1134(6)$ & $0.0590(4)$ & $0.0144(4)$ & $-0.0030(3)$ & $-0.0197(4)$ \\
C10 & $0.0480(14)$ & $0.0460(14)$ & $0.0432(13)$ & $0.0059(11)$ & $0.0087(11)$ & $0.0064(11)$ \\
C11 & $0.0540(15)$ & $0.0480(14)$ & $0.0428(13)$ & $0.0056(12)$ & $0.0066(11)$ & $0.0009(11)$ \\
N2 & $0.0459(12)$ & $0.0633(13)$ & $0.0525(12)$ & $0.0033(10)$ & $-0.0018(10)$ & $0.0017(10)$ \\
O1 & $0.0624(11)$ & $0.0998(14)$ & $0.0659(12)$ & $0.0237(10)$ & $0.0059(9)$ & $-0.0051(11)$ \\
C23 & $0.0431(14)$ & $0.0651(16)$ & $0.0504(14)$ & $-0.0048(12)$ & $-0.0039(11)$ & $0.0000(13)$ \\
N3 & $0.0499(12)$ & $0.0747(14)$ & $0.0498(12)$ & $0.0013(11)$ & $-0.0053(10)$ & $-0.0042(11)$ \\
N1 & $0.0583(13)$ & $0.0539(13)$ & $0.0571(13)$ & $0.0147(11)$ & $0.0091(10)$ & $0.0023(10)$ \\
C9 & $0.0499(14)$ & $0.0558(15)$ & $0.0401(13)$ & $0.0044(12)$ & $0.0069(11)$ & $0.0048(11)$ \\
C3 & $0.0498(15)$ & $0.0504(14)$ & $0.0479(13)$ & $0.0068(12)$ & $0.0084(11)$ & $0.0065(11)$ \\
C12 & $0.0471(14)$ & $0.0561(15)$ & $0.0460(13)$ & $0.0032(12)$ & $0.0035(11)$ & $0.0032(12)$ \\
C4 & $0.0533(15)$ & $0.0620(16)$ & $0.0423(13)$ & $0.0087(14)$ & $0.0051(11)$ & $0.0084(12)$ \\
C2 & $0.0667(17)$ & $0.0448(14)$ & $0.0584(15)$ & $0.0052(13)$ & $0.0043(13)$ & $-0.0011(12)$ \\
C24 & $0.0496(15)$ & $0.0677(17)$ & $0.0495(14)$ & $-0.0050(13)$ & $0.0039(12)$ & $-0.0042(12)$ \\
C18 & $0.0382(13)$ & $0.0596(15)$ & $0.0500(14)$ & $-0.0034(11)$ & $-0.0004(11)$ & $0.0039(12)$ \\
C19 & $0.0478(14)$ & $0.0759(17)$ & $0.0487(14)$ & $0.0004(13)$ & $0.0000(11)$ & $0.0013(13)$ \\
C16 & $0.0425(13)$ & $0.0601(15)$ & $0.0496(14)$ & $-0.0034(12)$ & $-0.0005(11)$ & $0.0004(12)$ \\
C20 & $0.0449(15)$ & $0.0738(17)$ & $0.0562(16)$ & $0.0009(13)$ & $0.0029(12)$ & $-0.0001(13)$ \\
C1 & $0.0579(16)$ & $0.0567(16)$ & $0.0559(15)$ & $-0.0036(13)$ & $0.0012(12)$ & $-0.0006(12)$ \\
C22 & $0.0516(15)$ & $0.0836(19)$ & $0.0533(15)$ & $0.0021(14)$ & $-0.0123(12)$ & $0.0033(14)$ \\
C8 & $0.0562(16)$ & $0.0674(17)$ & $0.0535(15)$ & $0.0046(13)$ & $0.0048(12)$ & $-0.0007(13)$ \\
C17 & $0.0450(14)$ & $0.0681(16)$ & $0.0463(14)$ & $-0.0043(13)$ & $-0.0052(11)$ & $0.0028(12)$ \\
C15 & $0.0510(15)$ & $0.0649(16)$ & $0.0542(15)$ & $0.0012(13)$ & $0.0058(12)$ & $-0.0031(13)$ \\
C6 & $0.0528(16)$ & $0.111(2)$ & $0.0561(16)$ & $0.0032(17)$ & $-0.0070(13)$ & $-0.0003(17)$ \\
C13 & $0.0713(17)$ & $0.0633(16)$ & $0.0612(16)$ & $0.0199(14)$ & $0.0127(14)$ & $-0.0010(14)$ \\
C21 & $0.0484(15)$ & $0.0732(17)$ & $0.0678(18)$ & $0.0049(13)$ & $-0.0062(13)$ & $0.0035(15)$ \\
& & & & & &
\end{tabular}




\begin{tabular}{lllllll} 
C5 & $0.0543(16)$ & $0.083(2)$ & $0.0564(16)$ & $0.0182(15)$ & $0.0018(13)$ & $0.0085(15)$ \\
C7 & $0.0610(18)$ & $0.083(2)$ & $0.0625(17)$ & $0.0000(16)$ & $-0.0006(14)$ & $-0.0098(14)$ \\
C25 & $0.083(2)$ & $0.117(3)$ & $0.0600(18)$ & $0.0211(18)$ & $0.0132(16)$ & $-0.0040(17)$ \\
C14 & $0.117(3)$ & $0.0603(18)$ & $0.080(2)$ & $0.0244(17)$ & $0.0234(18)$ & $0.0072(15)$ \\
\hline
\end{tabular}

Geometric parameters $\left(A,{ }^{o}\right)$

\begin{tabular}{|c|c|c|c|}
\hline $\mathrm{C} 11-\mathrm{C} 24$ & $1.752(2)$ & $\mathrm{C} 19-\mathrm{C} 20$ & $1.360(3)$ \\
\hline $\mathrm{C} 10-\mathrm{C} 11$ & $1.386(3)$ & C19-H19 & 0.9300 \\
\hline $\mathrm{C} 10-\mathrm{C} 3$ & $1.404(3)$ & $\mathrm{C} 16-\mathrm{C} 17$ & $1.369(3)$ \\
\hline $\mathrm{C} 10-\mathrm{C} 9$ & 1.449 (3) & $\mathrm{C} 16-\mathrm{C} 15$ & $1.458(3)$ \\
\hline $\mathrm{C} 11-\mathrm{C} 12$ & $1.382(3)$ & $\mathrm{C} 20-\mathrm{C} 21$ & $1.403(3)$ \\
\hline $\mathrm{C} 11-\mathrm{H} 11$ & 0.9300 & $\mathrm{C} 1-\mathrm{H} 1$ & 0.9300 \\
\hline $\mathrm{N} 2-\mathrm{C} 15$ & $1.269(3)$ & $\mathrm{C} 22-\mathrm{C} 21$ & $1.348(3)$ \\
\hline $\mathrm{N} 2-\mathrm{C} 12$ & $1.423(3)$ & $\mathrm{C} 22-\mathrm{H} 22$ & 0.9300 \\
\hline $\mathrm{O} 1-\mathrm{C} 20$ & $1.361(3)$ & $\mathrm{C} 8-\mathrm{C} 7$ & $1.378(3)$ \\
\hline $\mathrm{O} 1-\mathrm{C} 25$ & 1.425 & $\mathrm{C} 8-\mathrm{H} 8$ & 0.9300 \\
\hline $\mathrm{C} 23-\mathrm{N} 3$ & $1.361(3)$ & $\mathrm{C} 17-\mathrm{H} 17$ & 0.9300 \\
\hline $\mathrm{C} 23-\mathrm{C} 18$ & $1.409(3)$ & $\mathrm{C} 15-\mathrm{H} 15$ & 0.9300 \\
\hline $\mathrm{C} 23-\mathrm{C} 22$ & $1.413(3)$ & $\mathrm{C} 6-\mathrm{C} 5$ & $1.374(4)$ \\
\hline $\mathrm{N} 3-\mathrm{C} 24$ & $1.294(3)$ & $\mathrm{C} 6-\mathrm{C} 7$ & $1.382(4)$ \\
\hline $\mathrm{N} 1-\mathrm{C} 4$ & $1.378(3)$ & C6- $\mathrm{H} 6$ & 0.9300 \\
\hline $\mathrm{N} 1-\mathrm{C} 3$ & $1.380(3)$ & $\mathrm{C} 13-\mathrm{C} 14$ & $1.493(3)$ \\
\hline $\mathrm{N} 1-\mathrm{C} 13$ & $1.456(3)$ & $\mathrm{C} 13-\mathrm{H} 13 \mathrm{~A}$ & 0.9700 \\
\hline $\mathrm{C} 9-\mathrm{C} 8$ & $1.389(3)$ & $\mathrm{C} 13-\mathrm{H} 13 \mathrm{~B}$ & 0.9700 \\
\hline $\mathrm{C} 9-\mathrm{C} 4$ & $1.404(3)$ & $\mathrm{C} 21-\mathrm{H} 21$ & 0.9300 \\
\hline $\mathrm{C} 3-\mathrm{C} 2$ & $1.382(3)$ & $\mathrm{C} 5-\mathrm{H} 5$ & 0.9300 \\
\hline $\mathrm{C} 12-\mathrm{C} 1$ & $1.399(3)$ & $\mathrm{C} 7-\mathrm{H} 7$ & 0.9300 \\
\hline $\mathrm{C} 4-\mathrm{C} 5$ & $1.393(3)$ & $\mathrm{C} 25-\mathrm{H} 25 \mathrm{~A}$ & 0.9600 \\
\hline $\mathrm{C} 2-\mathrm{C} 1$ & $1.369(3)$ & $\mathrm{C} 25-\mathrm{H} 25 \mathrm{~B}$ & 0.9600 \\
\hline $\mathrm{C} 2-\mathrm{H} 2$ & 0.9300 & $\mathrm{C} 25-\mathrm{H} 25 \mathrm{C}$ & 0.9600 \\
\hline $\mathrm{C} 24-\mathrm{C} 16$ & $1.417(3)$ & $\mathrm{C} 14-\mathrm{H} 14 \mathrm{~A}$ & 0.9600 \\
\hline $\mathrm{C} 18-\mathrm{C} 17$ & $1.408(3)$ & C14-H14B & 0.9600 \\
\hline $\mathrm{C} 18-\mathrm{C} 19$ & $1.409(3)$ & $\mathrm{C} 14-\mathrm{H} 14 \mathrm{C}$ & 0.9600 \\
\hline $\mathrm{C} 11-\mathrm{C} 10-\mathrm{C} 3$ & $119.1(2)$ & $\mathrm{C} 2-\mathrm{C} 1-\mathrm{C} 12$ & $121.5(2)$ \\
\hline $\mathrm{C} 11-\mathrm{C} 10-\mathrm{C} 9$ & $134.5(2)$ & $\mathrm{C} 2-\mathrm{C} 1-\mathrm{H} 1$ & 119.2 \\
\hline $\mathrm{C} 3-\mathrm{C} 10-\mathrm{C} 9$ & $106.39(19)$ & $\mathrm{C} 12-\mathrm{C} 1-\mathrm{H} 1$ & 119.2 \\
\hline $\mathrm{C} 12-\mathrm{C} 11-\mathrm{C} 10$ & $119.8(2)$ & $\mathrm{C} 21-\mathrm{C} 22-\mathrm{C} 23$ & $121.0(2)$ \\
\hline $\mathrm{C} 12-\mathrm{C} 11-\mathrm{H} 11$ & 120.1 & $\mathrm{C} 21-\mathrm{C} 22-\mathrm{H} 22$ & 119.5 \\
\hline $\mathrm{C} 10-\mathrm{C} 11-\mathrm{H} 11$ & 120.1 & $\mathrm{C} 23-\mathrm{C} 22-\mathrm{H} 22$ & 119.5 \\
\hline $\mathrm{C} 15-\mathrm{N} 2-\mathrm{C} 12$ & $119.2(2)$ & $\mathrm{C} 7-\mathrm{C} 8-\mathrm{C} 9$ & $119.0(2)$ \\
\hline $\mathrm{C} 20-\mathrm{O} 1-\mathrm{C} 25$ & $117.2(2)$ & $\mathrm{C} 7-\mathrm{C} 8-\mathrm{H} 8$ & 120.5 \\
\hline $\mathrm{N} 3-\mathrm{C} 23-\mathrm{C} 18$ & $122.6(2)$ & $\mathrm{C} 9-\mathrm{C} 8-\mathrm{H} 8$ & 120.5 \\
\hline $\mathrm{N} 3-\mathrm{C} 23-\mathrm{C} 22$ & $119.6(2)$ & $\mathrm{C} 16-\mathrm{C} 17-\mathrm{C} 18$ & $121.7(2)$ \\
\hline $\mathrm{C} 18-\mathrm{C} 23-\mathrm{C} 22$ & $117.8(2)$ & $\mathrm{C} 16-\mathrm{C} 17-\mathrm{H} 17$ & 119.1 \\
\hline $\mathrm{C} 24-\mathrm{N} 3-\mathrm{C} 23$ & $117.3(2)$ & $\mathrm{C} 18-\mathrm{C} 17-\mathrm{H} 17$ & 119.1 \\
\hline $\mathrm{C} 4-\mathrm{N} 1-\mathrm{C} 3$ & $108.95(19)$ & $\mathrm{N} 2-\mathrm{C} 15-\mathrm{C} 16$ & $121.4(2)$ \\
\hline
\end{tabular}




\begin{tabular}{|c|c|c|c|}
\hline $\mathrm{C} 4-\mathrm{N} 1-\mathrm{C} 13$ & $125.7(2)$ & $\mathrm{N} 2-\mathrm{C} 15-\mathrm{H} 15$ & 119.3 \\
\hline $\mathrm{C} 3-\mathrm{N} 1-\mathrm{C} 13$ & $125.4(2)$ & $\mathrm{C} 16-\mathrm{C} 15-\mathrm{H} 15$ & 119.3 \\
\hline $\mathrm{C} 8-\mathrm{C} 9-\mathrm{C} 4$ & $119.6(2)$ & $\mathrm{C} 5-\mathrm{C} 6-\mathrm{C} 7$ & $122.5(3)$ \\
\hline $\mathrm{C} 8-\mathrm{C} 9-\mathrm{C} 10$ & $133.8(2)$ & $\mathrm{C} 5-\mathrm{C} 6-\mathrm{H} 6$ & 118.8 \\
\hline $\mathrm{C} 4-\mathrm{C} 9-\mathrm{C} 10$ & $106.5(2)$ & $\mathrm{C} 7-\mathrm{C} 6-\mathrm{H} 6$ & 118.8 \\
\hline $\mathrm{N} 1-\mathrm{C} 3-\mathrm{C} 2$ & $129.5(2)$ & $\mathrm{N} 1-\mathrm{C} 13-\mathrm{C} 14$ & $112.8(2)$ \\
\hline $\mathrm{N} 1-\mathrm{C} 3-\mathrm{C} 10$ & $109.1(2)$ & $\mathrm{N} 1-\mathrm{C} 13-\mathrm{H} 13 \mathrm{~A}$ & 109.0 \\
\hline $\mathrm{C} 2-\mathrm{C} 3-\mathrm{C} 10$ & $121.4(2)$ & $\mathrm{C} 14-\mathrm{C} 13-\mathrm{H} 13 \mathrm{~A}$ & 109.0 \\
\hline $\mathrm{C} 11-\mathrm{C} 12-\mathrm{C} 1$ & $119.7(2)$ & $\mathrm{N} 1-\mathrm{C} 13-\mathrm{H} 13 \mathrm{~B}$ & 109.0 \\
\hline $\mathrm{C} 11-\mathrm{C} 12-\mathrm{N} 2$ & $116.8(2)$ & $\mathrm{C} 14-\mathrm{C} 13-\mathrm{H} 13 \mathrm{~B}$ & 109.0 \\
\hline $\mathrm{C} 1-\mathrm{C} 12-\mathrm{N} 2$ & $123.5(2)$ & $\mathrm{H} 13 \mathrm{~A}-\mathrm{C} 13-\mathrm{H} 13 \mathrm{~B}$ & 107.8 \\
\hline $\mathrm{N} 1-\mathrm{C} 4-\mathrm{C} 5$ & $129.5(2)$ & $\mathrm{C} 22-\mathrm{C} 21-\mathrm{C} 20$ & $120.8(2)$ \\
\hline $\mathrm{N} 1-\mathrm{C} 4-\mathrm{C} 9$ & $109.0(2)$ & $\mathrm{C} 22-\mathrm{C} 21-\mathrm{H} 21$ & 119.6 \\
\hline $\mathrm{C} 5-\mathrm{C} 4-\mathrm{C} 9$ & $121.4(2)$ & $\mathrm{C} 20-\mathrm{C} 21-\mathrm{H} 21$ & 119.6 \\
\hline $\mathrm{C} 1-\mathrm{C} 2-\mathrm{C} 3$ & $118.3(2)$ & $\mathrm{C} 6-\mathrm{C} 5-\mathrm{C} 4$ & $117.1(2)$ \\
\hline $\mathrm{C} 1-\mathrm{C} 2-\mathrm{H} 2$ & 120.8 & $\mathrm{C} 6-\mathrm{C} 5-\mathrm{H} 5$ & 121.5 \\
\hline $\mathrm{C} 3-\mathrm{C} 2-\mathrm{H} 2$ & 120.8 & $\mathrm{C} 4-\mathrm{C} 5-\mathrm{H} 5$ & 121.5 \\
\hline $\mathrm{N} 3-\mathrm{C} 24-\mathrm{C} 16$ & $126.5(2)$ & $\mathrm{C} 8-\mathrm{C} 7-\mathrm{C} 6$ & $120.4(3)$ \\
\hline $\mathrm{N} 3-\mathrm{C} 24-\mathrm{C} 11$ & $115.38(18)$ & $\mathrm{C} 8-\mathrm{C} 7-\mathrm{H} 7$ & 119.8 \\
\hline $\mathrm{C} 16-\mathrm{C} 24-\mathrm{Cl1}$ & $118.17(19)$ & $\mathrm{C} 6-\mathrm{C} 7-\mathrm{H} 7$ & 119.8 \\
\hline $\mathrm{C} 17-\mathrm{C} 18-\mathrm{C} 23$ & $116.6(2)$ & $\mathrm{O} 1-\mathrm{C} 25-\mathrm{H} 25 \mathrm{~A}$ & 109.5 \\
\hline $\mathrm{C} 17-\mathrm{C} 18-\mathrm{C} 19$ & $123.2(2)$ & $\mathrm{O} 1-\mathrm{C} 25-\mathrm{H} 25 \mathrm{~B}$ & 109.5 \\
\hline $\mathrm{C} 23-\mathrm{C} 18-\mathrm{C} 19$ & $120.2(2)$ & $\mathrm{H} 25 \mathrm{~A}-\mathrm{C} 25-\mathrm{H} 25 \mathrm{~B}$ & 109.5 \\
\hline $\mathrm{C} 20-\mathrm{C} 19-\mathrm{C} 18$ & $119.9(2)$ & $\mathrm{O} 1-\mathrm{C} 25-\mathrm{H} 25 \mathrm{C}$ & 109.5 \\
\hline $\mathrm{C} 20-\mathrm{C} 19-\mathrm{H} 19$ & 120.1 & $\mathrm{H} 25 \mathrm{~A}-\mathrm{C} 25-\mathrm{H} 25 \mathrm{C}$ & 109.5 \\
\hline $\mathrm{C} 18-\mathrm{C} 19-\mathrm{H} 19$ & 120.1 & $\mathrm{H} 25 \mathrm{~B}-\mathrm{C} 25-\mathrm{H} 25 \mathrm{C}$ & 109.5 \\
\hline $\mathrm{C} 17-\mathrm{C} 16-\mathrm{C} 24$ & $115.2(2)$ & $\mathrm{C} 13-\mathrm{C} 14-\mathrm{H} 14 \mathrm{~A}$ & 109.5 \\
\hline $\mathrm{C} 17-\mathrm{C} 16-\mathrm{C} 15$ & $121.8(2)$ & $\mathrm{C} 13-\mathrm{C} 14-\mathrm{H} 14 \mathrm{~B}$ & 109.5 \\
\hline $\mathrm{C} 24-\mathrm{C} 16-\mathrm{C} 15$ & $122.9(2)$ & $\mathrm{H} 14 \mathrm{~A}-\mathrm{C} 14-\mathrm{H} 14 \mathrm{~B}$ & 109.5 \\
\hline $\mathrm{C} 19-\mathrm{C} 20-\mathrm{O} 1$ & $125.2(2)$ & $\mathrm{C} 13-\mathrm{C} 14-\mathrm{H} 14 \mathrm{C}$ & 109.5 \\
\hline $\mathrm{C} 19-\mathrm{C} 20-\mathrm{C} 21$ & $120.2(2)$ & $\mathrm{H} 14 \mathrm{~A}-\mathrm{C} 14-\mathrm{H} 14 \mathrm{C}$ & 109.5 \\
\hline $\mathrm{O} 1-\mathrm{C} 20-\mathrm{C} 21$ & $114.6(2)$ & $\mathrm{H} 14 \mathrm{~B}-\mathrm{C} 14-\mathrm{H} 14 \mathrm{C}$ & 109.5 \\
\hline $\mathrm{C} 3-\mathrm{C} 10-\mathrm{C} 11-\mathrm{C} 12$ & $-2.3(3)$ & $\mathrm{C} 22-\mathrm{C} 23-\mathrm{C} 18-\mathrm{C} 19$ & $2.0(3)$ \\
\hline $\mathrm{C} 9-\mathrm{C} 10-\mathrm{C} 11-\mathrm{C} 12$ & $176.1(2)$ & $\mathrm{C} 17-\mathrm{C} 18-\mathrm{C} 19-\mathrm{C} 20$ & $176.0(2)$ \\
\hline $\mathrm{C} 18-\mathrm{C} 23-\mathrm{N} 3-\mathrm{C} 24$ & $-2.7(3)$ & $\mathrm{C} 23-\mathrm{C} 18-\mathrm{C} 19-\mathrm{C} 20$ & $-1.4(4)$ \\
\hline $\mathrm{C} 22-\mathrm{C} 23-\mathrm{N} 3-\mathrm{C} 24$ & $177.2(2)$ & $\mathrm{N} 3-\mathrm{C} 24-\mathrm{C} 16-\mathrm{C} 17$ & $3.5(4)$ \\
\hline $\mathrm{C} 11-\mathrm{C} 10-\mathrm{C} 9-\mathrm{C} 8$ & $-0.4(4)$ & $\mathrm{C} 11-\mathrm{C} 24-\mathrm{C} 16-\mathrm{C} 17$ & $-176.18(17)$ \\
\hline $\mathrm{C} 3-\mathrm{C} 10-\mathrm{C} 9-\mathrm{C} 8$ & $178.2(2)$ & $\mathrm{N} 3-\mathrm{C} 24-\mathrm{C} 16-\mathrm{C} 15$ & $-173.0(2)$ \\
\hline $\mathrm{C} 11-\mathrm{C} 10-\mathrm{C} 9-\mathrm{C} 4$ & $-178.9(2)$ & $\mathrm{C} 11-\mathrm{C} 24-\mathrm{C} 16-\mathrm{C} 15$ & $7.3(3)$ \\
\hline $\mathrm{C} 3-\mathrm{C} 10-\mathrm{C} 9-\mathrm{C} 4$ & $-0.4(2)$ & $\mathrm{C} 18-\mathrm{C} 19-\mathrm{C} 20-\mathrm{O} 1$ & $-178.3(2)$ \\
\hline $\mathrm{C} 4-\mathrm{N} 1-\mathrm{C} 3-\mathrm{C} 2$ & $180.0(2)$ & $\mathrm{C} 18-\mathrm{C} 19-\mathrm{C} 20-\mathrm{C} 21$ & $-0.4(4)$ \\
\hline $\mathrm{C} 13-\mathrm{N} 1-\mathrm{C} 3-\mathrm{C} 2$ & $1.9(4)$ & $\mathrm{C} 25-\mathrm{O} 1-\mathrm{C} 20-\mathrm{C} 19$ & $-5.4(4)$ \\
\hline $\mathrm{C} 4-\mathrm{N} 1-\mathrm{C} 3-\mathrm{C} 10$ & $0.0(2)$ & $\mathrm{C} 25-\mathrm{O} 1-\mathrm{C} 20-\mathrm{C} 21$ & $176.6(2)$ \\
\hline $\mathrm{C} 13-\mathrm{N} 1-\mathrm{C} 3-\mathrm{C} 10$ & $-178.07(19)$ & $\mathrm{C} 3-\mathrm{C} 2-\mathrm{C} 1-\mathrm{C} 12$ & $-0.7(3)$ \\
\hline $\mathrm{C} 11-\mathrm{C} 10-\mathrm{C} 3-\mathrm{N} 1$ & $179.03(19)$ & $\mathrm{C} 11-\mathrm{C} 12-\mathrm{C} 1-\mathrm{C} 2$ & $-2.5(3)$ \\
\hline $\mathrm{C} 9-\mathrm{C} 10-\mathrm{C} 3-\mathrm{N} 1$ & $0.2(2)$ & $\mathrm{N} 2-\mathrm{C} 12-\mathrm{C} 1-\mathrm{C} 2$ & $176.4(2)$ \\
\hline $\mathrm{C} 11-\mathrm{C} 10-\mathrm{C} 3-\mathrm{C} 2$ & $-1.0(3)$ & $\mathrm{N} 3-\mathrm{C} 23-\mathrm{C} 22-\mathrm{C} 21$ & $179.4(2)$ \\
\hline
\end{tabular}




$\begin{array}{llll}\mathrm{C} 9-\mathrm{C} 10-\mathrm{C} 3-\mathrm{C} 2 & -179.8(2) & \mathrm{C} 18-\mathrm{C} 23-\mathrm{C} 22-\mathrm{C} 21 & -0.7(4) \\ \mathrm{C} 10-\mathrm{C} 11-\mathrm{C} 12-\mathrm{C} 1 & 4.0(3) & \mathrm{C} 4-\mathrm{C} 9-\mathrm{C} 8-\mathrm{C} 7 & 1.3(3) \\ \mathrm{C} 10-\mathrm{C} 11-\mathrm{C} 12-\mathrm{N} 2 & -174.97(19) & \mathrm{C} 10-\mathrm{C} 9-\mathrm{C} 8-\mathrm{C} 7 & -177.1(2) \\ \mathrm{C} 15-\mathrm{N} 2-\mathrm{C} 12-\mathrm{C} 11 & 150.7(2) & \mathrm{C} 24-\mathrm{C} 16-\mathrm{C} 17-\mathrm{C} 18 & -1.7(3) \\ \mathrm{C} 15-\mathrm{N} 2-\mathrm{C} 12-\mathrm{C} 1 & -28.2(3) & \mathrm{C} 15-\mathrm{C} 16-\mathrm{C} 17-\mathrm{C} 18 & 174.9(2) \\ \mathrm{C} 3-\mathrm{N} 1-\mathrm{C} 4-\mathrm{C} 5 & -178.0(2) & \mathrm{C} 23-\mathrm{C} 18-\mathrm{C} 17-\mathrm{C} 16 & -1.9(3) \\ \mathrm{C} 13-\mathrm{N} 1-\mathrm{C} 4-\mathrm{C} 5 & 0.0(4) & \mathrm{C} 19-\mathrm{C} 18-\mathrm{C} 17-\mathrm{C} 16 & -179.4(2) \\ \mathrm{C} 3-\mathrm{N} 1-\mathrm{C} 4-\mathrm{C} 9 & -0.2(2) & \mathrm{C} 12-\mathrm{N} 2-\mathrm{C} 15-\mathrm{C} 16 & -177.5(2) \\ \mathrm{C} 13-\mathrm{N} 1-\mathrm{C} 4-\mathrm{C} 9 & 177.83(19) & \mathrm{C} 17-\mathrm{C} 16-\mathrm{C} 15-\mathrm{N} 2 & -16.1(4) \\ \mathrm{C} 8-\mathrm{C} 9-\mathrm{C} 4-\mathrm{N} 1 & -178.43(19) & \mathrm{C} 24-\mathrm{C} 16-\mathrm{C} 15-\mathrm{N} 2 & 160.2(2) \\ \mathrm{C} 10-\mathrm{C} 9-\mathrm{C} 4-\mathrm{N} 1 & 0.4(2) & \mathrm{C} 4-\mathrm{N} 1-\mathrm{C} 13-\mathrm{C} 14 & 95.5(3) \\ \mathrm{C} 8-\mathrm{C} 9-\mathrm{C} 4-\mathrm{C} 5 & -0.4(3) & \mathrm{C} 3-\mathrm{N} 1-\mathrm{C} 13-\mathrm{C} 14 & -86.8(3) \\ \mathrm{C} 10-\mathrm{C} 9-\mathrm{C} 4-\mathrm{C} 5 & 178.4(2) & \mathrm{C} 23-\mathrm{C} 22-\mathrm{C} 21-\mathrm{C} 20 & -1.1(4) \\ \mathrm{N} 1-\mathrm{C} 3-\mathrm{C} 2-\mathrm{C} 1 & -177.5(2) & \mathrm{C} 19-\mathrm{C} 20-\mathrm{C} 21-\mathrm{C} 22 & 1.7(4) \\ \mathrm{C} 10-\mathrm{C} 3-\mathrm{C} 2-\mathrm{C} 1 & 2.5(3) & \mathrm{O} 1-\mathrm{C} 20-\mathrm{C} 21-\mathrm{C} 22 & 179.8(2) \\ \mathrm{C} 23-\mathrm{N} 3-\mathrm{C} 24-\mathrm{C} 16 & -1.4(4) & \mathrm{C} 7-\mathrm{C} 6-\mathrm{C} 5-\mathrm{C} 4 & 1.2(4) \\ \mathrm{C} 23-\mathrm{N} 3-\mathrm{C} 24-\mathrm{C} 11 & 178.33(17) & \mathrm{N} 1-\mathrm{C} 4-\mathrm{C} 5-\mathrm{C} 6 & 176.7(2) \\ \mathrm{N} 3-\mathrm{C} 23-\mathrm{C} 18-\mathrm{C} 17 & 4.2(3) & \mathrm{C} 9-\mathrm{C} 4-\mathrm{C} 5-\mathrm{C} 6 & -0.8(3) \\ \mathrm{C} 22-\mathrm{C} 23-\mathrm{C} 18-\mathrm{C} 17 & -175.7(2) & \mathrm{C} 9-\mathrm{C} 8-\mathrm{C} 7-\mathrm{C} 6 & -1.0(4) \\ \mathrm{N} 3-\mathrm{C} 23-\mathrm{C} 18-\mathrm{C} 19 & -178.1(2) & \mathrm{C} 5-\mathrm{C} 6-\mathrm{C} 7-\mathrm{C} 8 & -0.3(4) \\ \end{array}$

Hydrogen-bond geometry $\left(A,{ }^{\circ}\right)$

$\mathrm{Cg} 1$ and $\mathrm{Cg} 2$ are the centroids of the $\mathrm{C} 18-\mathrm{C} 23$ and $\mathrm{C} 1-\mathrm{C} 3 / \mathrm{C} 10-\mathrm{C} 12$ benzene rings, respectively.

\begin{tabular}{lllll}
\hline$D-\mathrm{H} \cdots A$ & $D-\mathrm{H}$ & $\mathrm{H} \cdots A$ & $D \cdots A$ & $D-\mathrm{H} \cdots A$ \\
\hline $\mathrm{C} 1-\mathrm{H} 1 \cdots C g 1^{\mathrm{i}}$ & 0.93 & 2.87 & $3.718(3)$ & 152 \\
$\mathrm{C} 7-\mathrm{H} 7 \cdots C g 2^{\mathrm{ii}}$ & 0.93 & 2.97 & $3.688(2)$ & 145 \\
\hline
\end{tabular}

Symmetry codes: (i) $-x,-y,-z+1$; (ii) $-x+1, y+1 / 2,-z+3 / 2$. 